\title{
Effect of color on rapidity gap survival
}

\author{
Zoltán Nagy ${ }^{*}$ \\ DESY, Notkestrasse 85, 22607 Hamburg, Germany \\ Davison E. Soper $\oplus^{\dagger}$ \\ Institute of Theoretical Science, University of Oregon, Eugene, Oregon 97403-5203, USA
}

(Received 21 May 2019; published 14 October 2019)

\begin{abstract}
We study the probability for no jets with transverse momenta above a given cut to be found in the rapidity region between two high $P_{\mathrm{T}}$ jets with a large rapidity separation. Our investigation uses the parton shower event generator DEDUCTOR with color beyond the leading-color-plus approximation included perturbatively.
\end{abstract}

DOI: 10.1103/PhysRevD.100.074012

\section{INTRODUCTION}

In the collision of two high energy protons, it can happen that two partons scatter with a fairly small angle but still with substantial transverse momenta. This produces two high $P_{\mathrm{T}}$ jets with a large difference in rapidity. At this Born level, there are no jets in the rapidity interval between the two high $P_{\mathrm{T}}$ jets. We say that there is a rapidity gap. Further QCD radiation can produce jets in the gap region, so that the rapidity gap does not survive.

In this paper, we investigate the role of color in the gap survival probability using the parton shower event generator DEDUCTOR [1-10], which incorporates a systematically improvable approximation with respect to QCD color [11].

To state the physical problem precisely, consider events in proton collisions at $\sqrt{s}=13 \mathrm{TeV}$. Using the anti- $k_{\mathrm{T}}$ jet algorithm [12] with a radius parameter $R$, find jets with transverse momenta $P_{\mathrm{T}}$ and rapidities $y$ with $-Y_{\text {cut }}<$ $y<Y_{\text {cut }}$. We will use $Y_{\text {cut }}=4.4$. Label the two highest $P_{\mathrm{T}}$ jets 1 and 2, with $y_{1}<y_{2}$. Define

$$
\begin{aligned}
\bar{p}_{\mathrm{T}} & =\frac{1}{2}\left(P_{\mathrm{T}, 1}+P_{\mathrm{T}, 2}\right), \\
y_{12} & =y_{2}-y_{1} .
\end{aligned}
$$

Now define a cut parameter $p_{\mathrm{T}}^{\text {cut }}$. We will take $p_{\mathrm{T}}^{\text {cut }}=$ $20 \mathrm{GeV}$. Look at those jets with $P_{\mathrm{T}}>p_{\mathrm{T}}^{\text {cut }}$ in the rapidity

\footnotetext{
ZZoltan.Nagy@desy.de

soper@uoregon.edu
}

Published by the American Physical Society under the terms of the Creative Commons Attribution 4.0 International license. Further distribution of this work must maintain attribution to the author(s) and the published article's title, journal citation, and DOI. Funded by SCOAP. region $y_{1}<y<y_{2}$ between the two leading jets. We will say that the event has a rapidity gap if there are no such jets in this rapidity region.

For given values of $\bar{p}_{\mathrm{T}}$ and $y_{12}$, let $f\left(\bar{p}_{\mathrm{T}}, y_{12}\right)$ be the fraction of events that have a gap. That is, $f$ is the ratio of the cross sections

$$
f\left(\bar{p}_{\mathrm{T}}, y_{12}\right)=\frac{d \sigma(\text { gap }) /\left[d \bar{p}_{\mathrm{T}} d y_{12}\right]}{d \sigma(\text { total }) /\left[d \bar{p}_{\mathrm{T}} d y_{12}\right]} .
$$

We can interpret $f$ as the probability that the gap survives after accounting for radiation beyond the Born level $2 \rightarrow 2$ scattering process. An alternative formulation is

$$
f\left(\bar{p}_{\mathrm{T}}, y_{12}\right)=1-\frac{d \sigma(\text { no gap }) /\left[d \bar{p}_{\mathrm{T}} d y_{12}\right]}{d \sigma(\text { total }) /\left[d \bar{p}_{\mathrm{T}} d y_{12}\right]} .
$$

Here $d \sigma$ (nogap) $/\left[d \bar{p}_{\mathrm{T}} d y_{12}\right]$ is the cross section to have the two gap-defining jets plus at least one more jet with $P_{\mathrm{T}}>p_{\mathrm{T}}^{\text {cut }}$ in the gap region. This formulation is useful for perturbative calculations because both the numerator and the denominator in the second term are infrared safe cross sections that can be calculated at next-to-leading order (NLO).

There is a practical reason to explore the calculation of the gap fraction $f$. In experimental investigations, it is often useful to look at some number of jets with transverse momenta $P_{\mathrm{T}} \sim Q$, where $Q$ is large, say hundreds of $\mathrm{GeV}$. These high $P_{\mathrm{T}}$ jets can be a signal of physics beyond the Standard Model and are the objects of primary interest. In order to reduce backgrounds, it may be useful to impose a requirement that there be no jets beyond this that have $P_{\mathrm{T}}$ greater than some value $p_{\mathrm{T}}^{\text {cut }}$, where $p_{\mathrm{T}}^{\text {cut }} \ll Q$. If one does this, one needs to be able to estimate the fraction of signal events with no extra jets and the fraction of background events with no extra jets. The calculation of these fractions involves potential large logarithms, $\log \left(Q / p_{\mathrm{T}}^{\text {cut }}\right)$. The large 
logarithms can spoil the usefulness of a calculation at a fixed order of perturbation theory. One can try to sum the large logarithms with an analytic calculation, but, as we suggest below, this is not entirely straightforward. An alternative is to use a parton shower event generator. This is the subject of this paper. The gap cross section defined above is the simplest example of a cross section that involves vetoing against extra jets.

There is also a motivation within QCD theory for examining the behavior of the gap fraction $f\left(\bar{p}_{\mathrm{T}}, y_{12}\right)$. In the case that $y_{12}$ is large, the behavior of $f$ as a function of $\bar{p}_{\mathrm{T}}$ and $y_{12}$ is a matter of substantial theoretical interest because it brings together several issues concerning the structure of QCD.

The perturbative expansion of the gap fraction $f$ contains two sorts of large logarithms. First, the logarithm $\log \left(\bar{p}_{\mathrm{T}} / p_{\mathrm{T}}^{\text {cut }}\right)$ can be large. Second, the rapidity separation $y_{12}$, which plays the role of a logarithm, can be large. At order $\alpha_{\mathrm{s}}^{N}$, a perturbative calculation can give us a factor of $\left[y_{12} \times \log \left(\bar{p}_{\mathrm{T}} / p_{\mathrm{T}}^{\text {cut }}\right)\right]^{N}$, so a summation of large logarithms is called for.

The summation of the large logarithms in $f$ is reviewed in Ref. [13]. In the simplest approximation for an analytic summation of leading logarithms [14,15], one uses the exponential of a Sudakov exponent constructed from the one loop graphs for the virtual exchange of a low transverse momentum gluon. However, the analytic treatment is not straightforward [16], so that a complete analytic summation is not available. The logarithms to be summed are "nonglobal" in that emissions into the gap region count differently from emissions outside of the gap region [17-20]. There are imaginary contributions, in which a factor $y_{12}$ is replaced by a factor $i \pi$. Furthermore, in some contributions, a factor of $y_{12}$ or $\mathrm{i} \pi$ becomes a factor of $\log \left(\bar{p}_{\mathrm{T}} / p_{\mathrm{T}}^{\text {cut }}\right)$ [13,21-23].

An alternative to an analytical summation of the large logarithms associated with $f$ is the use of a parton shower event generator like DEDUCTOR, which we use in this paper. We expect this to be useful because the splitting functions used in such a generator reflect the soft and collinear singularities that lead to the large logarithms. Furthermore, a parton shower treatment conserves momentum exactly at each step, whereas analytic treatments sometimes neglect the momentum of soft gluons.

One can worry that factors of $y_{12}$ that arise from integrating emissions over a range $y_{1}<y<y_{2}$ may not be properly generated in a hardness ordered shower like DEDUCTOR if emitted gluons have roughly the same transverse momentum $P_{\mathrm{T}}$. Perhaps it would be better to use a shower based on evolution in rapidity like HEJ $[24,25]$. However, with the ordering variable $\Lambda^{2}$ that is the default in DEDUCTOR and is used in this paper, it is possible to have successive gluon emissions with similar $P_{\mathrm{T}}$ values if the rapidities $y$ of the emitted gluons are very different. This kinematic feature is discussed in some detail in Ref. [6].
The partons in the developing event radiate because they carry QCD color. In the very simplest approximation, the probability for gluon radiation from a quark is proportional to $C_{\mathrm{F}}=\left(N_{\mathrm{c}}^{2}-1\right) /\left(2 N_{\mathrm{c}}\right)$ and the probability for gluon radiation from a gluon is proportional to $C_{\mathrm{A}}=N_{\mathrm{c}}$, where $N_{\mathrm{c}}=3$ is the number of colors. In a somewhat more sophisticated calculation, one can calculate emission probabilities in the leading color (LC) approximation, keeping contributions of leading order in an expansion in powers of $1 / N_{\mathrm{c}}^{2}$. However, it is not self-evident that the leading color approximation is adequate for such a calculation. For instance, the perturbative expansion of the gap fraction can contain terms of the form $\left(1 / N_{\mathrm{c}}^{2}\right)\left[\alpha_{\mathrm{s}} y_{12} \log \left(\bar{p}_{\mathrm{T}} / p_{\mathrm{T}}^{\text {cut }}\right)\right]^{N}$. If we do not work beyond leading color, we lose such contributions.

In order to study the effect on the gap fraction of color beyond the leading color approximation, we use DEDUCTOR. The base color approximation in DEDUCTOR is the LC+ approximation [4], which includes some contributions that are suppressed by factors of $1 / N_{\mathrm{c}}^{2}$. The effects of using the LC+ approximation are described in Ref. [8]. With the current version ${ }^{1}$ of DEDUCTOR, we can go beyond the LC + approximation. As explained in Ref. [11], the operator that generates parton splittings with exactly the color content dictated by QCD Feynman diagrams is an operator denoted $\mathcal{H}_{I}(t)$. In the $\mathrm{LC}+$ approximation, $\mathcal{H}_{I}(t)$ is approximated by an operator $\mathcal{H}^{\mathrm{LC}+}(t)$ that has a simpler color structure. To get from the LC+ approximation to full color for splittings, we need another operator, $\Delta \mathcal{H}(t)$, defined by

$$
\mathcal{H}_{I}(t)=\mathcal{H}^{\mathrm{LC}+}(t)+\Delta \mathcal{H}(t)
$$

Similarly, the operator that generates approximate virtual graphs in a shower with exactly the color content dictated by QCD Feynman diagrams is an operator denoted $\mathcal{V}(t)$. This operator is used to construct the Sudakov factor for each shower step. In the LC + approximation, $\mathcal{V}(t)$ is approximated by an operator $\mathcal{V}^{\mathrm{LC}+}(t)$. To get to full color for the virtual diagrams, we need another operator, $\Delta \mathcal{V}(t)$, defined by

$$
\mathcal{V}(t)=\mathcal{V}^{\mathrm{LC}+}(t)+\Delta \mathcal{V}(t)
$$

The added contribution $\Delta \mathcal{V}(t)$ includes an operator that contains a factor $i \pi$ :

$$
\Delta \mathcal{V}(t)=\Delta \mathcal{V}_{\operatorname{Re}}(t)+\mathcal{V}_{\mathrm{i} \pi}(t)
$$

Now, DEDUCTOR allows one to include as many powers of $\Delta \mathcal{H}(t)$ and $\Delta \mathcal{V}(t)$ as one wants, within practical limits.

\footnotetext{
${ }^{1}$ Version 3.0.3 of the code, used in this paper, is available at http://www.desy.de/znagy/deductor/ and http://pages.uoregon .edu/soper/deductor/.
} 
In the calculations in this paper, we first investigate how many powers we need and then use just that number.

The commonly used parton shower algorithms PYTHIA [26], HERWIG [27], and SHERPA [28] work in the leading color approximation. There has been work other than ours on extending the accuracy of parton shower algorithms beyond the leading color approximation. Reference [29] works with color amplitudes, as in [11], but does not account for collinear singularities and so far lacks a parton shower implementation. References [30-32] treat $\Delta \mathcal{H}(t)$, but not $\Delta \mathcal{V}(t)$.

This paper is structured as follows. There are three sections with preparatory information: Sec. II about putting the events in bins in $\bar{p}_{\mathrm{T}}$, Sec. III about DEDUCTOR, and Sec. IV about how many powers of $\Delta \mathcal{H}(t)$ and $\Delta \mathcal{V}(t)$ we need. Then Sec. V contains the results on the gap fraction $f$ as a function of $\bar{p}_{\mathrm{T}}$ and $y_{12}$. This includes results about the dependence of $f$ on the jet size parameter $R$, a comparison of the parton shower calculation to a purely perturbative calculation, and a comparison to results from PYTHIA. Finally, Sec. VI presents some conclusions.

\section{PUTTING THE CALCULATION IN BINS}

The gap fraction is a function $f\left(\bar{p}_{\mathrm{T}}, y_{12}\right)$ of the average transverse momentum of the jets that define the gap region and of their rapidity difference. It is defined by

$$
f\left(\bar{p}_{\mathrm{T}}, y_{12}\right)=\frac{d \sigma(\text { gap }) /\left[d \bar{p}_{\mathrm{T}} d y_{12}\right]}{d \sigma(\text { total }) /\left[d \bar{p}_{\mathrm{T}} d y_{12}\right]} .
$$

We organize the calculation of $f$ in bins of $\bar{p}_{\mathrm{T}}$ and $y_{12}$ : $P_{i}<\bar{p}_{\mathrm{T}}<P_{i+1}, Y_{n}<y_{12}<Y_{n+1}$. For each bin, the ratio that we calculate is

$$
\begin{aligned}
f= & {\left[\int_{Y_{n}}^{Y_{n+1}} d y_{12} \int_{P_{i}}^{P_{i+1}} d \bar{p}_{\mathrm{T}} \frac{d \sigma(\text { gap })}{d \bar{p}_{\mathrm{T}} d y_{12}} \frac{1}{h\left(n, \bar{p}_{\mathrm{T}}\right)}\right] / } \\
& {\left[\int_{Y_{n}}^{Y_{n+1}} d y_{12} \int_{P_{i}}^{P_{i+1}} d \bar{p}_{\mathrm{T}} \frac{d \sigma(\text { total })}{d \bar{p}_{\mathrm{T}} d y_{12}} \frac{1}{h\left(n, \bar{p}_{\mathrm{T}}\right)}\right] . }
\end{aligned}
$$

Here the function $h\left(n, \bar{p}_{\mathrm{T}}\right)$ is chosen so that $\left(1 / h\left(n, \bar{p}_{\mathrm{T}}\right)\right) d \sigma($ total $) /\left[d \bar{p}_{\mathrm{T}} d y_{12}\right]$ is approximately constant inside the bin. This gives us

$$
f=\int_{Y_{n}}^{Y_{n+1}} d y_{12} \int_{P_{i}}^{P_{i+1}} d \bar{p}_{\mathrm{T}} f\left(\bar{p}_{\mathrm{T}}, y_{12}\right) w\left(\bar{p}_{\mathrm{T}}, y_{12}\right),
$$

where the weight factor $w$ is

$$
w\left(\bar{p}_{\mathrm{T}}, y_{12}\right)=\frac{1}{N} \frac{d \sigma(\text { total })}{d \bar{p}_{\mathrm{T}} d y_{12}} \frac{1}{h\left(n, \bar{p}_{\mathrm{T}}\right)},
$$

with

$$
N=\int_{Y_{n}}^{Y_{n+1}} d y_{12} \int_{P_{i}}^{P_{i+1}} d \bar{p}_{\mathrm{T}} \frac{d \sigma(\text { total })}{d \bar{p}_{\mathrm{T}} d y_{12}} \frac{1}{h\left(n, \bar{p}_{\mathrm{T}}\right)},
$$

so that the integral of $w$ over the bin equals 1.

\section{THE DEDUCTOR SHOWER}

Our analysis is based on the parton shower event generator, DEDUCTOR [1-11]. In this section, we review some of the features of DEDUCTOR that are particularly relevant to the gap survival problem.

The shower begins after a leading order $2 \rightarrow 2$ hard scattering. It would be desirable to use a next-to-leading order hard scattering with matching to the shower, but this option is not yet available in DEDUCTOR. For the hard scattering, we choose renormalization and factorization scales $\mu_{R}=\mu_{\mathrm{F}}=P_{\mathrm{T}}^{\mathrm{Born}} / \sqrt{2}$, where $P_{\mathrm{T}}^{\text {Born }}$ is the transverse momentum in the Born scattering that initiates the shower.

We use the default shower ordering variable in DEDUCTOR, $\Lambda$, which is based on virtuality. For massless partons, the definition is

$$
\begin{aligned}
\Lambda^{2} & =\frac{\left(\hat{p}_{l}+\hat{p}_{m+1}\right)^{2}}{2 p_{l} \cdot Q_{0}} Q_{0}^{2} \text { final state, } \\
\Lambda^{2} & =\frac{\left|\left(\hat{p}_{\mathrm{a}}-\hat{p}_{m+1}\right)^{2}\right|}{2 p_{\mathrm{a}} \cdot Q_{0}} Q_{0}^{2} \text { initial state. }
\end{aligned}
$$

Here the mother parton in a final state splitting has momentum $p_{l}$ and the daughters have momenta $\hat{p}_{l}$ and $\hat{p}_{m+1}$. For an initial state splitting in hadron A, the mother parton has momentum $p_{\mathrm{a}}$, the new (in backward evolution) initial state parton has momentum $\hat{p}_{\mathrm{a}}$ and the final state parton created in the splitting has momentum $\hat{p}_{m+1}$. We denote by $Q_{0}$ a fixed vector equal to the total momentum of all of the final state partons just after the hard scattering that initiates the shower. The motivation for this choice is described in Ref. [6].

Successive splittings have $\Lambda_{n+1}<\Lambda_{n}$. For the first splitting, we demand that $\Lambda$ be smaller than a chosen shower start scale, $\mu_{\mathrm{s}}$. We choose

$$
\mu_{\mathrm{s}}=\frac{3}{2} P_{\mathrm{T}}^{\mathrm{Born}} .
$$

This choice is motivated in Ref. [10]. Results do not change much if we choose a larger value for $\mu_{\mathrm{s}}$ because the DEDUCTOR splitting kernel restricts the splitting transverse momentum to be no greater than $P_{\mathrm{T}}^{\mathrm{Born}}$ in order to ensure that the scattering that initiates the shower is the highest $P_{\mathrm{T}}$ scattering in the event.

The treatment of color in DEDUCTOR is described in detail in Ref. [11].

The base color treatment in DEDUCTOR is the $\mathrm{LC}+$ approximation [4]. The LC+ approximation produces terms whose contributions are suppressed by powers $1 / N_{\mathrm{c}}^{n}$, 
where $N_{\mathrm{c}}=3$ is the number of colors. There is no need to carry terms suppressed by large powers of $1 / N_{c}$, so we impose a maximum value on the color suppression index $I$ associated with a partonic color state [4]. Contributions to cross sections with a given value $I$ of the color suppression index come with a factor $1 / N_{\mathrm{c}}^{n}$ with $n \geq I$. Thus we can neglect contributions with large values of $I$. We choose a value for a parameter $I_{\max }$. In this paper, we choose $I_{\max }=4$. The shower operator switches its behavior if it reaches a value of $I$ with $I-I_{\text {hard }} \geq I_{\max }$, were $I_{\text {hard }}$ is the color suppression index of the hard scattering state at the start of the shower. First, the shower switches to an approximate shower based on the color group $\mathrm{U}\left(N_{\mathrm{c}}\right)$ instead of $\mathrm{SU}\left(N_{\mathrm{c}}\right)$. Second, splittings that would increase $I$ are not allowed. Thus contributions proportional to $1 / N_{\mathrm{c}}^{I_{\max }}$ are calculated only approximately.

The LC+ approximation is a substantial improvement over the leading color (LC) approximation, but it still leaves a lot out. What it leaves out are color operators $\Delta \mathcal{H}, \Delta \mathcal{V}_{\operatorname{Re}}$ and $\mathcal{V}_{\mathrm{i} \pi}$ from Eqs. (4), (5) and (6) [11]. A calculation that included all powers of these operators would be exact in color. DEDUCTOR cannot do that, but it can include a user-specified maximum number of powers of $\Delta \mathcal{H}, \Delta \mathcal{V}_{\mathrm{Re}}$ and $\mathcal{V}_{\mathrm{i} \pi}$. Here $\Delta \mathcal{H}$ is the part of parton splitting graphs that is omitted in the LC+ approximation; $\Delta \mathcal{V}_{\mathrm{Re}}$ is the real part of approximated virtual graphs omitted in the $\mathrm{LC}+$ approximation; and $\mathcal{V}_{\mathrm{i} \pi}$ is the imaginary part of virtual graphs, which contain a factor $i \pi$.

The user controls the level of approximation by specifying integers $N_{\Delta}^{\mathrm{thr}}, N_{\mathrm{Re}}$, and $N_{\mathrm{i} \pi}$.

First, just after the hard scattering, DEDUCTOR inserts an operator $\mathcal{U}_{\mathcal{V}}$ that produces a summation of threshold logarithms [10]. The operator $\mathcal{U}_{\mathcal{V}}$ gives results as an expansion in powers of $\Delta \mathcal{V}_{\mathrm{Re}}$. DEDUCTOR retains only those terms with no more than $N_{\Delta}^{\text {thr }}$ factors of $\Delta \mathcal{V}_{\mathrm{Re}}$.

Second, the operators $\Delta \mathcal{H}, \Delta \mathcal{V}_{\mathrm{Re}}$ and $\mathcal{V}_{\mathrm{i} \pi}$ appear in the shower evolution operator $\mathcal{U}\left(t_{2}, t_{1}\right)$. The operator $\mathcal{U}\left(t_{2}, t_{1}\right)$ produces terms proportional to $[\Delta \mathcal{H}]^{A}\left[\Delta \mathcal{V}_{\mathrm{Re}}\right]^{B}\left[\mathcal{V}_{\mathrm{i} \pi}\right]^{C}$. DEDUCTOR retains only terms with $A+B \leq N_{\mathrm{Re}}, C \leq N_{\mathrm{i} \pi}$, and $A+B+C \leq \max \left\{N_{\mathrm{Re}}, N_{\mathrm{i} \pi}\right\}$.

Finally, if the shower evolution reaches a state in which the color suppression index $I$ has $I-I_{\text {hard }} \geq I_{\max }$, then the evolution omits any further contributions from $\Delta \mathcal{H}, \Delta \mathcal{V}_{\operatorname{Re}}$ and $\mathcal{V}_{\mathrm{i} \pi}$ and switches to a $\mathrm{U}\left(N_{\mathrm{c}}\right)$ instead of an $\mathrm{SU}\left(N_{\mathrm{c}}\right)$ shower, while not allowing $I$ to increase further.

DEDUCTOR allows the user to specify $N_{\Delta}^{\text {thr }}$ at the start of the parton shower. Then the user can specify approximation parameters $N_{\mathrm{Re}}, N_{\mathrm{i} \pi}$ and $I_{\max }$ for an evolution interval $\mu_{\mathrm{s}}>\Lambda>\Lambda(1)$ and then smaller parameters for successive following evolution intervals $\Lambda(i)>\Lambda>\Lambda(i+1)$. In this paper, we use parameters $N_{\mathrm{Re}}, N_{\mathrm{i} \pi}$ and $I_{\max }=4$ for the interval $\mu_{\mathrm{s}}>\Lambda>\Lambda(1)=30 \mathrm{GeV}$. Then we either stop the shower at $30 \mathrm{GeV}$ or continue it to $\Lambda(2)=1 \mathrm{GeV}$ with the LC + approximation, $N_{\mathrm{Re}}=N_{\mathrm{i} \pi}=0$, with, still, $I_{\max }=4$.
The DEDUCTOR splitting kernel also limits the transverse momenta $k_{\mathrm{T}}$ in splittings to be larger than $k_{\mathrm{T}}^{\mathrm{min}} \approx 1 \mathrm{GeV}$. The precise value is $k_{\mathrm{T}}^{\mathrm{min}}=1.0 \mathrm{GeV}$ for final state splittings and $k_{\mathrm{T}}^{\min }=1.295 \mathrm{GeV}$, set by the starting scale of the parton distributions that we use, for initial state splittings.

\section{EFFECT OF ORDER OF APPROXIMATION}

As outlined above, we apply limits on the accuracy of the parton shower calculation by specifying parameters $N_{\Delta}^{\text {thr }}$, $N_{\mathrm{Re}}, N_{\mathrm{i} \pi}$ and $I_{\max }$, along with the range of the hardness variable $\Lambda$ over which the parameters apply. In this section, we try to estimate the systematic error in the calculation that results from these limits.

For the color suppression index, we set $I_{\max }=4$. This means that contributions to $f$ that carry a factor $1 / N_{\mathrm{c}}^{4} \approx$ $10^{-2}$ and beyond are calculated only approximately. Thus we estimate a systematic error on $f$ of \pm 0.01 from not taking a larger value of $I_{\max }$.

For our studies of the gap fraction, in the evolution range $\mu_{\mathrm{s}}>\Lambda>30 \mathrm{GeV}$, we will choose $N_{\mathrm{Re}}=2, N_{\mathrm{i} \pi}=2$, and $N_{\Delta}^{\text {thr }}=1$. How much systematic error should one ascribe to not choosing larger values to these parameters? We investigate that question in this section by changing these parameters one at a time, while leaving the remaining parameters set to zero. In this investigation, we examine $f$ in the range $300 \mathrm{GeV}<\bar{p}_{\mathrm{T}}<400 \mathrm{GeV}$ and $4<y_{12}<5$. We set $N_{\mathrm{Re}}$ and $N_{\mathrm{i} \pi}$ in the evolution range $\mu_{\mathrm{s}}>\Lambda>$ $30 \mathrm{GeV}$ and stop evolution at $\Lambda=30 \mathrm{GeV}$.

We first investigate how $f$ depends on $N_{\text {Re }}$, with $N_{\Delta}^{\mathrm{thr}}=N_{\mathrm{i} \pi}=0$. The results are shown in Fig. 1 . The value of $f$ for $N_{\mathrm{Re}}=0$ is the result of a calculation in the $\mathrm{LC}+$ approximation. We see that adding one factor of $\Delta \mathcal{H}$ or $\Delta \mathcal{V}_{\operatorname{Re}}$ changes $f$ by $-0.041 \pm 0.004$, approximately a $20 \%$ change. Choosing $N_{\mathrm{Re}}=2$ leads to a further change of $+0.028 \pm 0.010$. We expect that increasing $N_{\mathrm{Re}}$ beyond 2 will change $f$ by less than 0.02 . Indeed, we find that choosing $N_{\mathrm{Re}}=3$ leads to a further change of $0.01 \pm 0.02$. Based on this study, we estimate that the error on $f$ from limiting $N_{\operatorname{Re}}$ to 2 is \pm 0.02 .

We next investigate how $f$ depends on $N_{\mathrm{i} \pi}$ with $N_{\mathrm{Re}}=$ $N_{\Delta}^{\mathrm{thr}}=0$. The results are shown in Fig. 2. Only even powers of $\mathcal{V}_{\mathrm{i} \pi}$ contribute to the cross section since $\mathcal{V}_{\mathrm{i} \pi}$ has a factor i. Thus we show results only for $N_{\mathrm{i} \pi}=0,2$, and 4 . We see that increasing $N_{\mathrm{i} \pi}$ from 0 to 2 in $\mathcal{U}$ raises the gap fraction by $0.016 \pm 0.002$. Adding two more powers of $\mathcal{V}_{\mathrm{i} \pi}$ leaves the gap fraction unchanged, within the statistical error. The change is $0.000 \pm 0.003$. We conclude that $N_{\mathrm{i} \pi}=2$ is a reasonable choice and that \pm 0.01 is a reasonable error estimate for the effect of added factors of $\mathcal{V}_{\mathrm{i} \pi}$ beyond 2 .

We next investigate how $f$ depends on $N_{\Delta}^{\text {thr }}$ with $N_{\mathrm{Re}}=N_{\mathrm{i} \pi}=0$. The results are shown in Fig. 3. We see that increasing $N_{\Delta}^{\text {thr }}$ from 0 to 1 lowers the gap fraction by $0.011 \pm 0.002$. Increasing $N_{\Delta}^{\text {thr }}$ above 1 has hardly any 


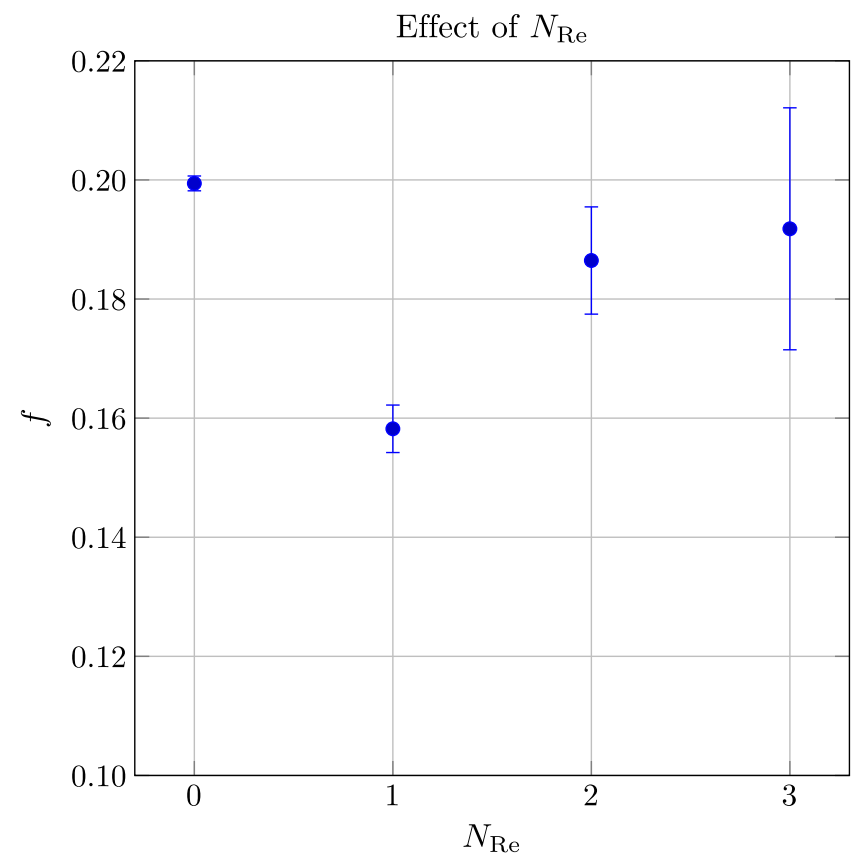

FIG. 1. Gap fraction $f$ in the range $300 \mathrm{GeV}<\bar{p}_{\mathrm{T}}<400 \mathrm{GeV}$ and $4<y_{12}<5$ calculated with different values of $N_{\mathrm{Re}}$ with $N_{\mathrm{i} \pi}=N_{\Delta}^{\mathrm{thr}}=0$.

effect. We conclude that $N_{\Delta}^{\text {thr }}=1$ is a reasonable choice and that \pm 0.01 is a reasonable error estimate for the effect of added color in the threshold operator in this calculation.

In the calculations of the gap fraction in the following section, we use $N_{\mathrm{Re}}=2, N_{\mathrm{i} \pi}=2, N_{\Delta}^{\mathrm{thr}}=1$ in the range $\mu_{\mathrm{s}}>\Lambda>\Lambda_{\min }$, where we choose $\Lambda_{\min }=30 \mathrm{GeV}$. Then

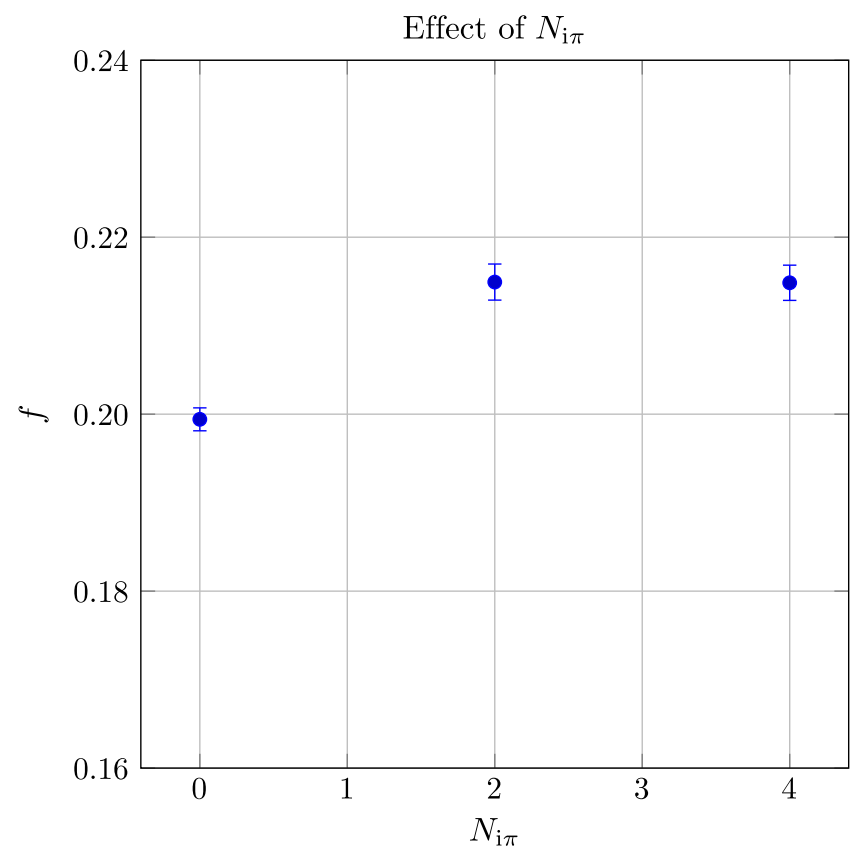

FIG. 2. Gap fraction $f$ as in Fig. 1 calculated with different values of $N_{\mathrm{i} \pi}$ with $N_{\mathrm{Re}}=N_{\Delta}^{\mathrm{thr}}=0$.

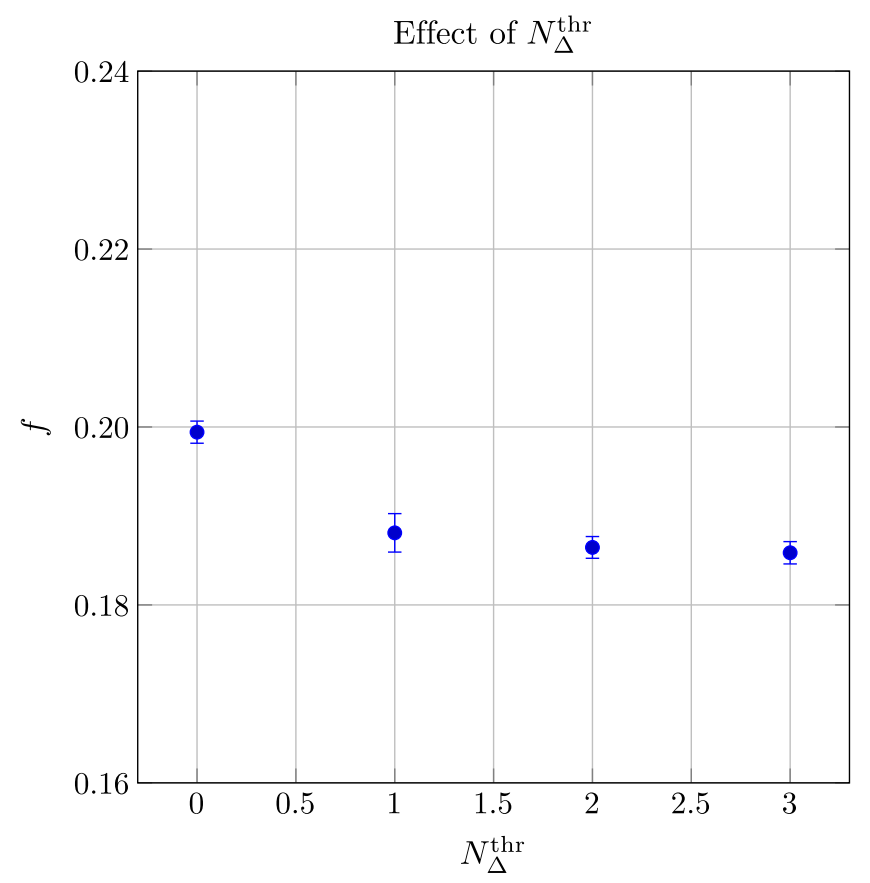

FIG. 3. Gap fraction $f$ as in Fig. 1 calculated with different values of $N_{\Delta}^{\mathrm{thr}}$ with $N_{\mathrm{Re}}=N_{\mathrm{i} \pi}=0$.

we revert to the LC+ approximation for $\Lambda_{\min }>\Lambda>$ $1 \mathrm{GeV}$. The range $\mu_{\mathrm{s}}>\Lambda>30 \mathrm{GeV}$ covers a range of about a factor of 10 or more in $\Lambda$ for $\bar{p}_{\mathrm{T}}>200 \mathrm{GeV}$ (with $\mu_{\mathrm{s}}=3 \bar{p}_{\mathrm{T}} / 2$ ). We hope that this range is adequate to

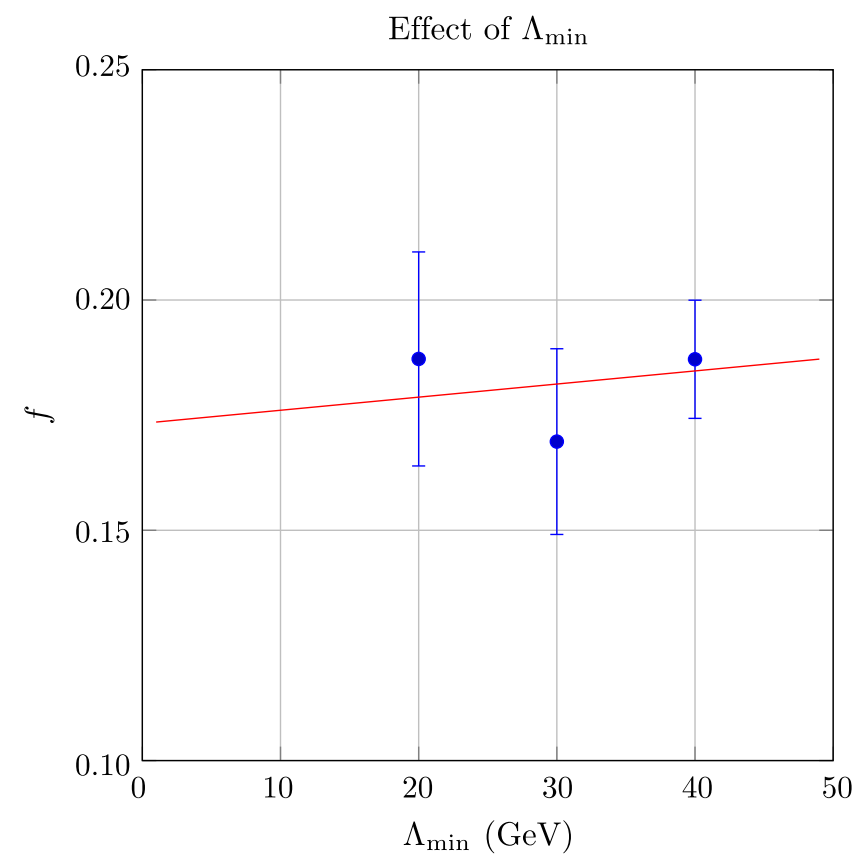

FIG. 4. Gap fraction $f$ in the range $300 \mathrm{GeV}<\bar{p}_{\mathrm{T}}<400 \mathrm{GeV}$ and $4<y_{12}<5$ as a function of $\Lambda_{\min }$. The calculation uses $N_{\Delta}^{\text {thr }}=1$ at the start of the shower, then $N_{\mathrm{Re}}=2, N_{\mathrm{i} \pi}=2$ for the shower in the range $\mu_{\mathrm{s}}>\Lambda>\Lambda_{\min }$, then $N_{\mathrm{Re}}=N_{\mathrm{i} \pi}=0$ in the range $\Lambda_{\min }>\Lambda>1 \mathrm{GeV}$. The maximum color suppression index is $I_{\max }=4$. The curve is a linear fit to the numerical results. 
explore the effect of extra color contributions. However, we will find that including color beyond the $\mathrm{LC}+$ approximation generally makes $f$ smaller. Thus if we were able to include the extra color contributions in the range $30 \mathrm{GeV}>\Lambda>1 \mathrm{GeV}$, presumably the calculated $f$ would be somewhat smaller. If we were to simply set $\Lambda_{\min }=$ $1 \mathrm{GeV}$, DEDUCTOR would generate very complicated color states in a large number of quite soft splittings, so that few events would result and the statistical fluctuations in $f$ would be large. An alternative is to try to estimate the effect of leaving $\Lambda_{\min }=30 \mathrm{GeV}$ by extrapolating in $\Lambda_{\min }$. We calculate $f$ in the range $300 \mathrm{GeV}<\bar{p}_{\mathrm{T}}<400 \mathrm{GeV}$ and $4<\Delta y<5$ for $\Lambda_{\min }=20 \mathrm{GeV}, 30 \mathrm{GeV}$ and $40 \mathrm{GeV}$. If we assume a linear model, $f=a_{0}+a_{1} \Lambda_{\min } /(30 \mathrm{GeV})$, we can fit $a_{0}$ and $a_{1}$. Then results for $f$ in this $\bar{p}_{\mathrm{T}}$ and $y_{12}$ range calculated with $\Lambda_{\min }=30 \mathrm{GeV}$ should be corrected by subtracting $a_{1}$ from $f$. The results for $f$ and the fit are shown in Fig. 4. We find $a_{1}=0.01 \pm 0.04$. That is, $a_{1}$ equals 0 within its statistical error. However, with the accuracy obtained for $a_{1}$, we have an extrapolation error on $f$ in this $\bar{p}_{\mathrm{T}}$ and $y_{12}$ range of about \pm 0.04 .

\section{RESULTS FOR THE GAP FRACTION}

We are now ready to look at the results for the gap fraction $f$. The jets are defined with the anti- $k_{\mathrm{T}}$ algorithm. We start with radius parameter $R=0.4$. Then we will examine how $f$ depends on $R$. We choose five different bins for $y_{12}$ and examine $f$ in each bin as a function of $\bar{p}_{\mathrm{T}}$.

We use $N_{\mathrm{Re}}=2, N_{\mathrm{i} \pi}=2, N_{\Delta}^{\mathrm{thr}}=1$ and $I_{\max }=4$. There are systematic errors in the results that arise from not using larger values of $N_{\mathrm{Re}}, N_{\mathrm{i} \pi}, N_{\Delta}^{\mathrm{thr}}$ and $I_{\max }$. In Sec. IV, we estimated these systematic errors in $f$ at about \pm 0.02 .

In each case, the results were obtained with the stated values of $N_{\operatorname{Re}}$ and $N_{\mathrm{i} \pi}$ in the shower between $\Lambda=\mu_{\mathrm{s}}$ and $\Lambda=\Lambda_{\min }=30 \mathrm{GeV}$. For the rest of the shower, down to $\Lambda=1 \mathrm{GeV}$, we used the LC+ approximation. There is a systematic error from not using a smaller value of $\Lambda_{\min }$, based on how well we could extrapolate to $\Lambda_{\min }=1 \mathrm{GeV}$. In Sec. IV, we estimate this extrapolation error at \pm 0.04 .

There are also systematic errors from not having shower splitting functions beyond order $\alpha_{\mathrm{s}}$ and from starting the shower with just lowest order parton scattering. We do not estimate these systematic errors and, rather, regard the results as an investigation of color effects within a calculation at this order of approximation.

Finally, there are statistical errors from the fluctuations in Monte Carlo event generation. The statistical errors are rather substantial for the largest values of $y_{12}$ and $\bar{p}_{\mathrm{T}}$. We do not exhibit error bands that represent the statistical errors since the size of the fluctuations is evident in the differences of $f$ between neighboring values of $\bar{p}_{\mathrm{T}}$. DEDUCTOR, of course, provides an estimated error for each bin, but our impression is that these estimated errors are somewhat smaller than the bin to bin fluctuations.

We now turn to the results.

\section{A. Gap fraction for $\boldsymbol{R}=\mathbf{0 . 4}$}

We begin with results for jets defined with the anti- $k_{\mathrm{T}}$ algorithm with a radius parameter $R=0.4$, shown in Fig. 5 . We choose five different bins for $y_{12}$ and examine $f\left(\bar{p}_{\mathrm{T}}\right)$ as a function of $\bar{p}_{\mathrm{T}}$. In each $y_{12}$ bin, we show three curves, all obtained with $I_{\max }=4$. The first, in blue, is obtained with just the LC+ approximation. Then, in green, we show results obtained with contributions from $\Delta \mathcal{H}$ and $\Delta \mathcal{V}_{\mathrm{Re}}$

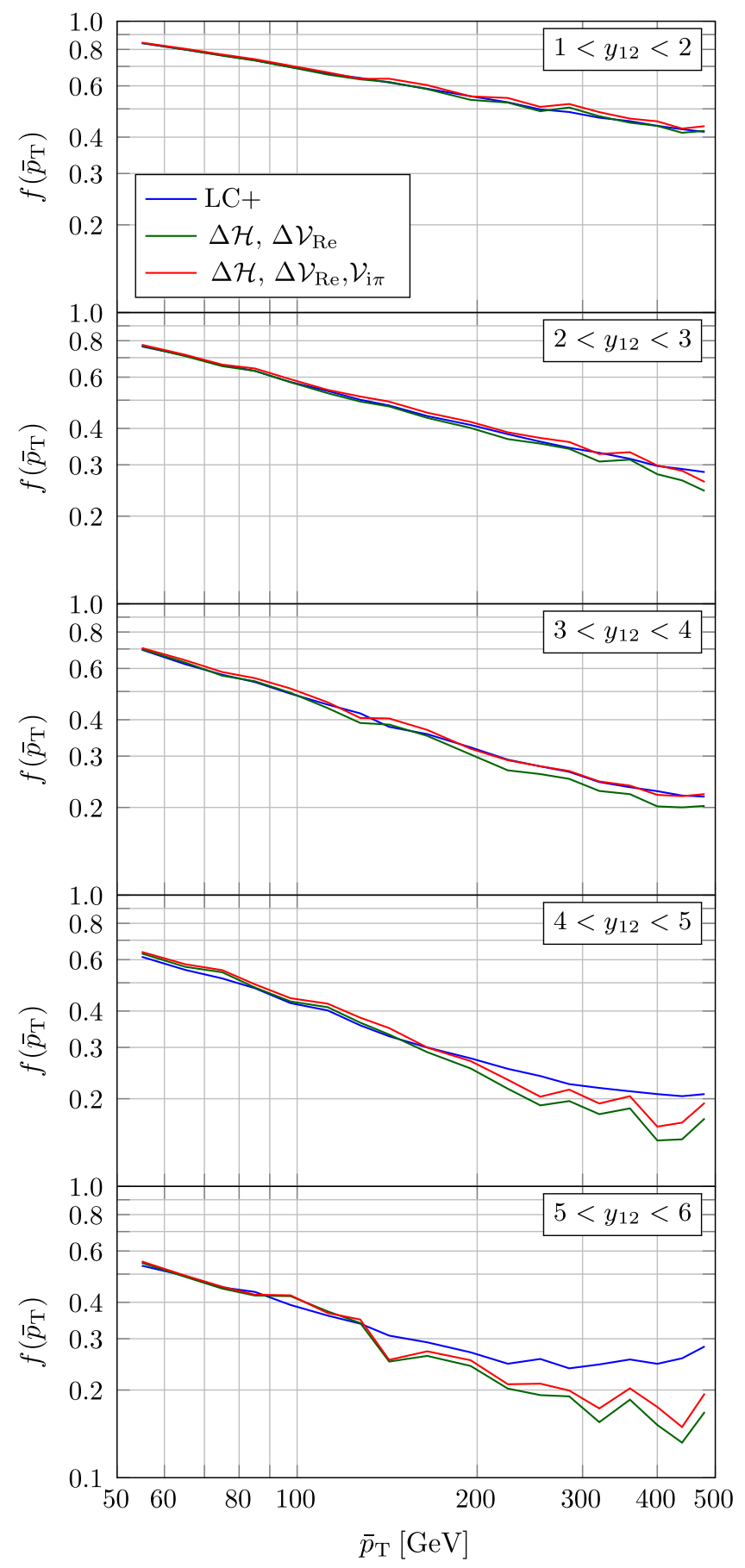

FIG. 5. Gap fraction $f$ for $R=0.4$ versus $y_{12}$ and $\bar{p}_{\mathrm{T}}$. 
using $N_{\operatorname{Re}}=2$ and $N_{\Delta}^{\text {thr }}=1$. Here contributions from $\mathcal{V}_{\mathrm{i} \pi}$ are omitted. Finally, in red, we show results obtained with contributions from all of $\Delta \mathcal{H}, \Delta \mathcal{V}_{\mathrm{Re}}$ and $\mathcal{V}_{\mathrm{i} \pi}$ using $N_{\mathrm{Re}}=2$, $N_{\mathrm{i} \pi}=2$, and $N_{\Delta}^{\mathrm{thr}}=1$.

Look first at $f\left(\bar{p}_{\mathrm{T}}\right)$ for $1<y_{12}<2$. We see that all three plots are almost straight lines. That is, $f$ has the approximate form

$$
f\left(\bar{p}_{\mathrm{T}}\right) \approx \exp \left(-A_{0}-A_{1} \log \left(\bar{p}_{\mathrm{T}} / p_{\mathrm{T}}^{\text {cut }}\right)\right) .
$$

We find that $A_{1} \approx 0.33$. It appears, then, that the dependence of $f$ on the large logarithm $\log \left(\bar{p}_{\mathrm{T}} / p_{\mathrm{T}}^{\text {cut }}\right)$ exponentiates. We also note that the three curves are almost identical: the contributions from the operators $\Delta \mathcal{H}, \Delta \mathcal{V}_{\operatorname{Re}}$ and $\mathcal{V}_{\mathrm{i} \pi}$ are quite small.

Look next at $f\left(\bar{p}_{\mathrm{T}}\right)$ for $2<y_{12}<3$. We see that all three plots are almost straight lines. The slope of the lines is larger: for $\mathrm{LC}+, A_{1} \approx 0.50$. This is what we expect. There is now a larger gap to radiate gluons into, so it is easier for the gap not to survive.

We also see that the contributions from $\Delta \mathcal{H}$ and $\Delta \mathcal{V}_{\operatorname{Re}}$ have begun to matter. Including the effects of these operators multiplies $f$ by a factor of about $\exp (-0.13)$ in the region $\bar{p}_{\mathrm{T}}>300 \mathrm{GeV}$. Some effect like this was to be expected. The operator $\Delta \mathcal{H}$ gives soft gluon emission in the angular region between two emitting partons, one in the ket state and one in the bra state. Such a gluon can destroy the gap. There is now a wider angular region for this to happen, so it is not unexpected that an effect of $\Delta \mathcal{H}$ and $\Delta \mathcal{V}_{\operatorname{Re}}$ would begin to be visible.

The contribution from $\mathcal{V}_{\mathrm{i} \pi}^{2}$ is visible as the difference between the red and green curves in Fig. 5. We see that for $2<y_{12}<3$, the contribution from $\mathcal{V}_{\mathrm{i} \pi}^{2}$ has begun to matter. The operator $\mathcal{V}_{\mathrm{i} \pi}$ does not create any final state gluons. However, it changes the color state of the color density matrix. With different color, the probability for the other operators to emit gluons into the gap can change. Thus, with a wider gap, it is not a surprise that $\mathcal{V}_{\mathrm{i} \pi}$ now has a visible effect.

What is surprising, at least to us, is that the effect from $\mathcal{V}_{\mathrm{i} \pi}$ has about the same magnitude as the effect from $\Delta \mathcal{H}$ and $\Delta \mathcal{V}_{\operatorname{Re}}$, but has the opposite sign. Thus when we add the effects together, we are almost back to the LC + curve.

Now look at $f\left(\bar{p}_{\mathrm{T}}\right)$ for $3<y_{12}<4$. For $\bar{p}_{\mathrm{T}}<200 \mathrm{GeV}$, the $\mathrm{LC}+$ curve is quite precisely a straight line, but now with a larger slope: $A_{1} \approx 0.61$, continuing the previous trend.

The effect of $\Delta \mathcal{H}$ and $\Delta \mathcal{V}_{\operatorname{Re}}$ in the region $\bar{p}_{\mathrm{T}}>380 \mathrm{GeV}$ has now grown very slightly. The effect of $\mathcal{V}_{\mathrm{i} \pi}$ still approximately cancels the effect of $\Delta \mathcal{H}$ and $\Delta \mathcal{V}_{\operatorname{Re}}$.

There is a new effect that is now visible. Beyond $\bar{p}_{\mathrm{T}} \approx 200 \mathrm{GeV}$, the LC + curve is no longer a good fit to a straight line. Rather, it curves up slightly. One could

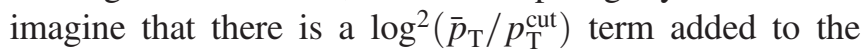
exponent in Eq. (14). However, a fit to the results for
$\bar{p}_{\mathrm{T}}<200 \mathrm{GeV}$ that includes a $\log ^{2}\left(\bar{p}_{\mathrm{T}} / p_{\mathrm{T}}^{\text {cut }}\right)$ contribution still gives very nearly a straight line, which does not fit the results for $\bar{p}_{\mathrm{T}}>200 \mathrm{GeV}$. Thus we have a nonlogarithmic large $\bar{p}_{\mathrm{T}}$ effect. Such an effect is to be expected because of what we might call momentum starvation. If the two jets that define the gap have equal transverse momenta, then the c.m. energy of the colliding partons that could scatter to make these jets is $[\hat{s}]^{1 / 2}=\bar{p}_{\mathrm{T}} \exp \left(y_{12} / 2\right)$. For $\bar{p}_{\mathrm{T}}=$ $400 \mathrm{GeV}$ and $y_{12}=3.5$, this is $[\hat{s}]^{1 / 2}=2.3 \mathrm{TeV}$. Since parton distribution functions fall with momentum fraction $x$, it is quite improbable to have a parton collision with this much $[\hat{s}]^{1 / 2}$. It is then more improbable to have an even larger $[\hat{s}]^{1 / 2}$ needed to radiate a gluon with enough transverse momentum to destroy the gap. Thus the gap fraction $f\left(\bar{p}_{\mathrm{T}}\right)$ should be larger than it would be if this effect were ignored. Furthermore, this effect should become more pronounced as $y_{12}$ increases.

The fact that a momentum starvation effect is visible in the DEDUCTOR results indicates that momentum conservation is important in the calculation.

Most of the trends that we have observed for $y_{12}<4$ continue for $4<y_{12}<5$ and $5<y_{12}<6$.

For $\bar{p}_{\mathrm{T}}<200 \mathrm{GeV}$, the $\mathrm{LC}+$ curves are still quite precisely straight lines. However, the slopes do not grow with $y_{12}$. For the regions $3<y_{12}<4,4<y_{12}<5$ and $5<y_{12}<6$ we have, respectively, $A_{1} \approx 0.61, A_{1} \approx 0.65$ and $A_{1} \approx 0.56$.

As we expect, the upward turn of the $\mathrm{LC}+$ curve for $\bar{p}_{\mathrm{T}}>200 \mathrm{GeV}$ becomes more pronounced as $y_{12}$ increases.

The effect of including $\Delta \mathcal{H}$ and $\Delta \mathcal{V}_{\mathrm{Re}}$ grows as $y_{12}$ increases. In the region $\bar{p}_{\mathrm{T}}>300 \mathrm{GeV}$, This effect multiplies $f$ by a factor of about $\exp (-0.41)$ for $4<y_{12}<5$ and $\exp (-0.81)$ for $5<y_{12}<6$.

The previous trend of an increasing effect from $\mathcal{V}_{\mathrm{i} \pi}$ does not continue. The effect from including $\mathcal{V}_{\mathrm{i} \pi}$ is to multiply $f$ in the region $\bar{p}_{\mathrm{T}}>300 \mathrm{GeV}$ by a factor of about $\exp (+0.14)$ for $3<y_{12}<4$, $\exp (+0.18)$ for $4<y_{12}<5$, and $\exp (+0.14)$ for $5<y_{12}<6$. Thus the $\mathcal{V}_{\mathrm{i} \pi}$ effect does not cancel the growing effect of $\Delta \mathcal{H}$ and $\Delta \mathcal{V}_{\mathrm{Re}}$.

In principle, there should be contributions to $f$ proportional to $\mathcal{V}_{\mathrm{i} \pi}^{2}$ that contain an extra power of $\log \left(\bar{p}_{\mathrm{T}} / p_{\mathrm{T}}^{\text {cut }}\right)$, dubbed a "superleading log" [13,21-23]. These contributions are surely present, but they are not large enough to be visible in the difference between the red and green curves in Fig. 5.

We are left with a net decrease in $f$ for $\bar{p}_{\mathrm{T}}>300 \mathrm{GeV}$ from color beyond the $\mathrm{LC}+$ approximation by a factor of about $\exp (-0.23)$ for $4<y_{12}<5$ and $\exp (-0.61)$ for $5<y_{12}<6$.

\section{B. Gap fraction dependence on $\boldsymbol{R}$}

How does the choice of the cone size parameter $R$ affect the gap fraction? To find out, we carried out the previous calculation also for $R=0.2$ and $R=0.7$. We then divided 
$f[R=0.2]$ by $f[R=0.7]$ for each $y_{12}$ range and for each $\bar{p}_{\mathrm{T}}$. The extent to which $f[R=0.2] / f[R=0.7]$ differs from 1 indicates the influence of $R$ on $f$. The results are plotted in Fig. 6.

We see that when $y_{12}$ is not too large the gap fraction is smaller for $R=0.2$ than it is for $R=0.7$. This is easy to understand. There is a high probability to emit a gluon near the direction of one of the two leading jets that defines ends

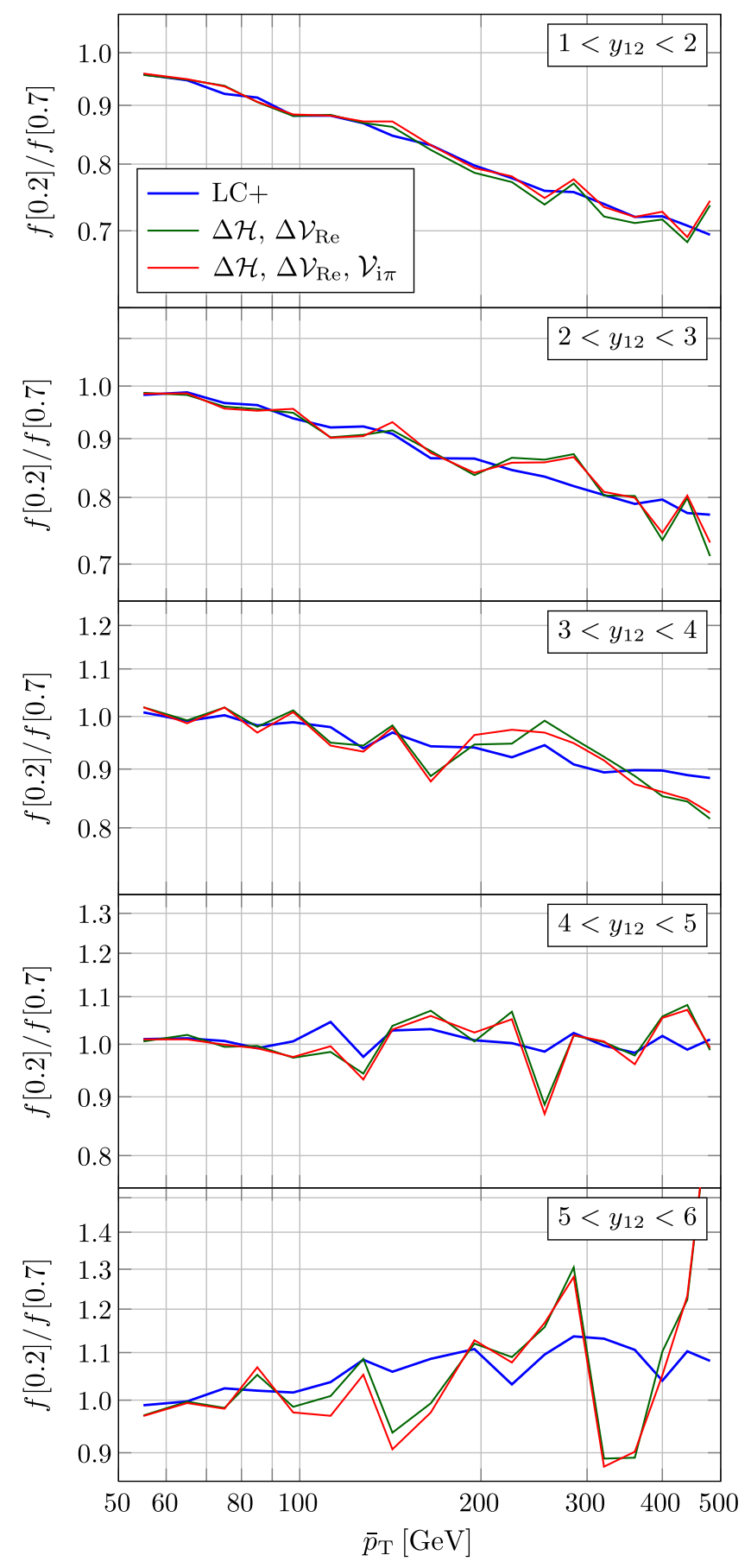

FIG. 6. Gap fraction ratio $f[R=0.2] / f[R=0.7]$ versus $y_{12}$ and $\bar{p}_{\mathrm{T}}$. of the gap region. For a large jet radius $R$, this gluon is likely to form part of the jet. But for small $R$, this gluon can fall outside of the jet but inside the gap region, thus destroying the gap. That is, roughly collinear gluon radiation will decrease the gap fraction when $R$ is small.

This effect of decreasing $f$ with decreasing $R$ diminishes as $y_{12}$ grows. This trend is easy to understand because for large $y_{12}$ there is a wide range for emission of a gluon that will destroy the gap, so that the range near the two jets that define the gap region is not so important.

We note that for $5<y_{12}<6$ and $\bar{p}_{\mathrm{T}}>100 \mathrm{GeV}$, the gap fraction increases with decreasing $R$. This is not a large effect, but it is striking because it reverses the expected trend that we see for smaller $y_{12}$.

How do the extra color operators $\Delta \mathcal{H}$ and $\Delta \mathcal{V}$ affect the $R$ dependence of the gap fraction distribution? We see from Fig. 6 that there is no effect within the statistical errors. At the simplest level, this is easy to understand. The real emission operator $\Delta \mathcal{H}$ reflects singularities for emission of soft gluons in directions that are not particularly close to the directions of existing partons. It does not contain collinear singularities. However, $R$ dependence for single emissions is connected with collinear singularities. Now, $R$ dependence could arise from a collinear emission followed by a wide angle soft emission, so we could see some $R$ dependence coming from $\Delta \mathcal{H}$ and $\Delta \mathcal{V}$. However, it is not a surprise that this dependence is small.

\section{Gap fraction in perturbation theory}

We can calculate the gap fraction in fixed order perturbation theory instead of using a parton shower. We write the gap fraction in the form of Eq. (3). Here $d \sigma$ (total)/ $\left[d \bar{p}_{\mathrm{T}} d y_{12}\right]$ is the cross section to produce at least two $R=$ 0.4 jets in the rapidity window $-4.4<y<4.4$ such that the two jets in the rapidity window with the largest $P_{\mathrm{T}}$ satisfy $\bar{p}_{\mathrm{T}}=\left(P_{\mathrm{T}, 1}+P_{\mathrm{T}, 2}\right) / 2$ and $y_{12}=\left|y_{1}-y_{2}\right|$. This is an infrared safe jet cross section for which the lowest order contribution has two partons in the final state. The cross section $d \sigma$ (nogap) $/\left[d \bar{p}_{\mathrm{T}} d y_{12}\right]$ is the cross section to produce at least three $R=0.4$ jets in the rapidity window $-4.4<y<4.4$ such that the two jets in the rapidity window with the largest $P_{\mathrm{T}}$ satisfy $\bar{p}_{\mathrm{T}}=\left(P_{\mathrm{T}, 1}+P_{\mathrm{T}, 2}\right) / 2$ and $y_{12}=\left|y_{1}-y_{2}\right|$ and such that there is a third jet with $\min \left(y_{1}, y_{2}\right)<y_{3}<\max \left(y_{1}, y_{2}\right)$ and $P_{\mathrm{T}, 3}>p_{\mathrm{T}}^{\text {cut }}=20 \mathrm{GeV}$. This is an infrared safe jet cross section for which the lowest order contribution has three partons in the final state. We calculate both $d \sigma$ (total) $/\left[d \bar{p}_{\mathrm{T}} d y_{12}\right]$ and $d \sigma$ (no gap)/ $\left[d \bar{p}_{\mathrm{T}} d y_{12}\right]$ at NLO using NLOJET++ [33]. For these perturbative calculations, our primary choice for the factorization and renormalization scales is $\mu_{\mathrm{F}}=\mu_{\mathrm{R}}=2 \bar{p}_{\mathrm{T}}$. We investigate the scale dependence by also using $\mu_{\mathrm{F}}=\mu_{\mathrm{R}}=\bar{p}_{\mathrm{T}}$ and $\mu_{\mathrm{F}}=\mu_{\mathrm{R}}=4 \bar{p}_{\mathrm{T}}$.

In Ref. [10], we calculated the gap fraction in this manner for $\sqrt{s}=7 \mathrm{TeV}$. We found that, although there was substantial dependence on the scale choice for large 
$y_{12}$, the perturbative calculation with $\mu_{\mathrm{F}}=\mu_{\mathrm{R}}=2 \bar{p}_{\mathrm{T}}$ worked quite well. This was surprising to us because there are large logarithms that are not summed in the perturbative calculation. In this paper, we have chosen $\sqrt{s}=13 \mathrm{TeV}$. Now there is a larger range available for gluon emissions.

Figure 7, we show the perturbative results for $\sqrt{s}=13 \mathrm{TeV}$. For each range of $y_{12}$, we show a central curve with $\mu_{\mathrm{F}}=\mu_{\mathrm{R}}=2 \bar{p}_{\mathrm{T}}$. We show how the result varies

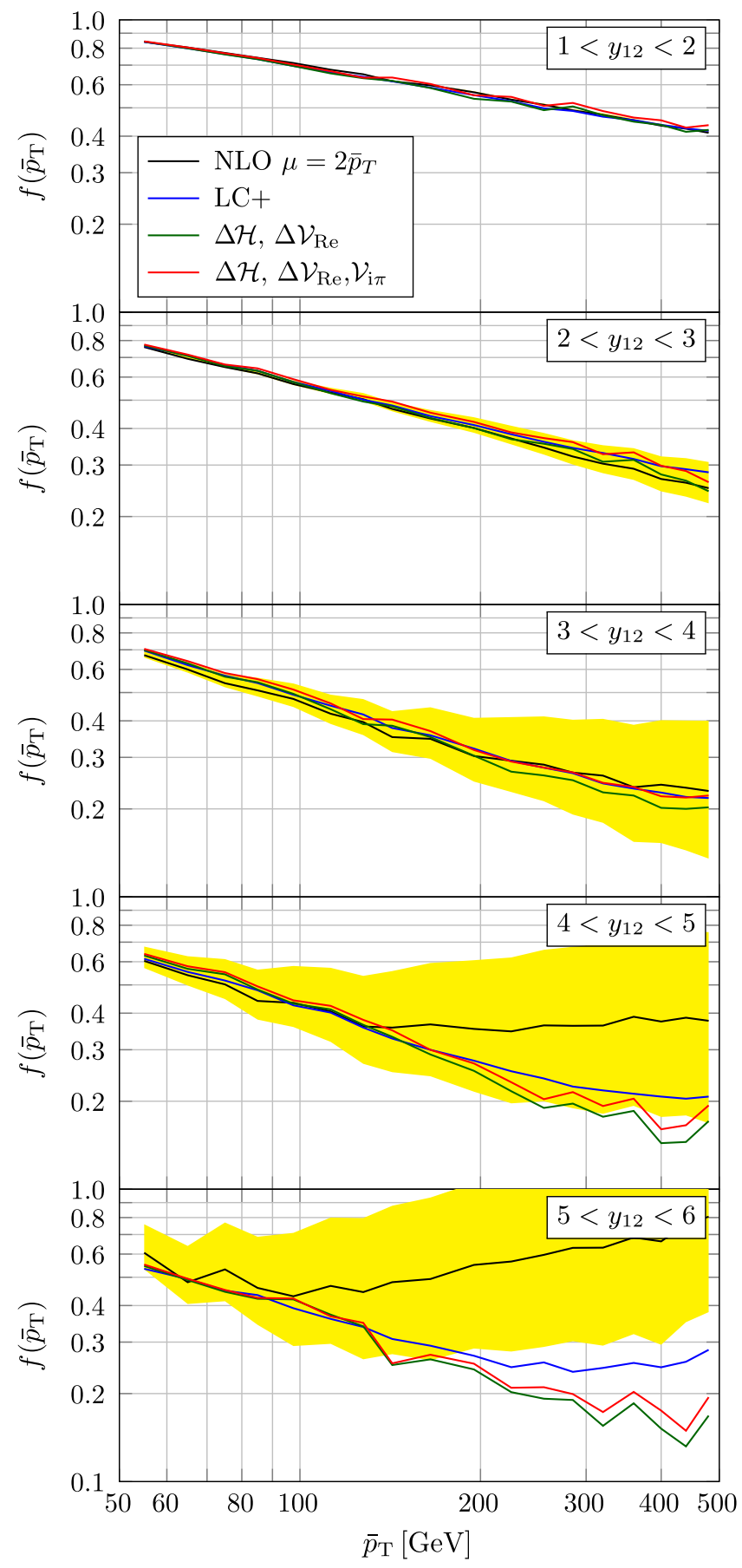

FIG. 7. Gap fraction calculated perturbatively to NLO using $\mu_{\mathrm{R}}=\mu_{\mathrm{F}}=2 \bar{p}_{\mathrm{T}}$, with an error band for $\bar{p}_{\mathrm{T}}<\mu_{\mathrm{R}}, \mu_{\mathrm{F}}<4 \bar{p}_{\mathrm{T}}$. The DEDUCTOR curves from Fig. 5 are also shown. for $\bar{p}_{\mathrm{T}}<\mu_{\mathrm{F}}=\mu_{\mathrm{R}}<4 \bar{p}_{\mathrm{T}}$ as a yellow error band. We also show the DEDUCTOR results from Fig. 5. We see that the perturbative results for $f$ agree with the DEDUCTOR results within about $20 \%$ for $1<y_{12}<2$ and $2<y_{12}<3$. For $3<y_{12}<4$, the agreement between the two types of calculation is still good, but the scale variation error band on the perturbative calculation has grown substantially. For $4<y_{12}<5$ and $5<y_{12}<6$, the scale variation error

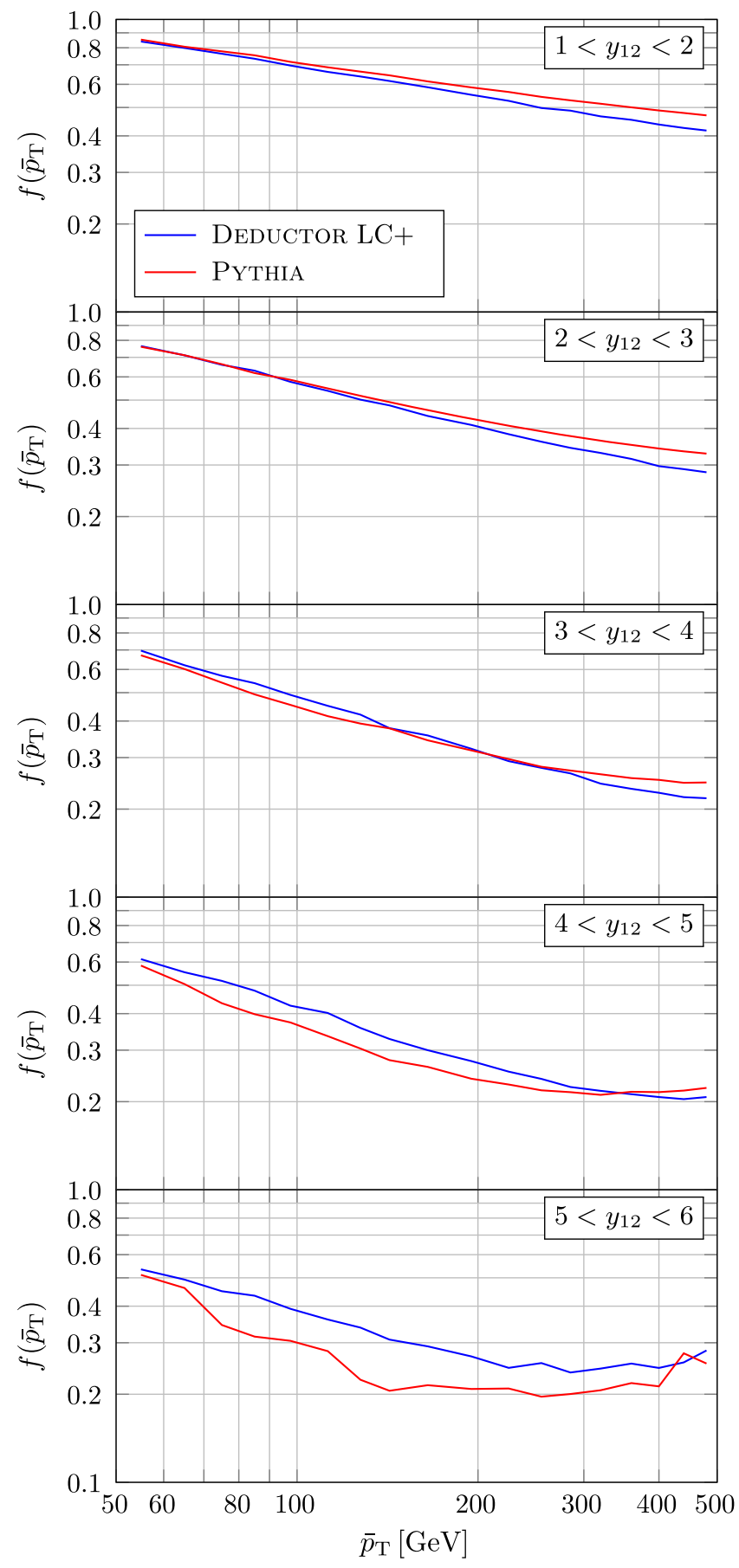

FIG. 8. Gap fraction $f$ for $R=0.4$ versus $y_{12}$ and $\bar{p}_{\mathrm{T}}$ for PYTHIA and for DEDUCTOR with the LC + approximation. 
band is so large that one can conclude that the NLO perturbative calculation is not reliable. Thus one needs either a parton shower calculation or an analytic summation of the large logarithms.

\section{Gap fraction in PYTHIA}

We can also compare the gap fraction results from DEDUCTOR with the analogous results from PYTHIA [26]. Since PYTHIA is limited to the leading color approximation, we compare to DEDUCTOR with the LC+ approximation. Our previous investigations [10] have indicated that nonperturbative effects are quite small for the gap fraction, so we have not included any nonperturbative effects in the results from DEDUCTOR in this paper. ${ }^{2}$ Accordingly, we have not included the underlying event and hadronization in PYTHIA. We used PYTHIA 8.423 with default settings except that we set $\alpha_{\mathrm{s}}\left(M_{Z}^{2}\right)=0.118$ in the shower and include the "CMW" factor in the $\alpha_{\mathrm{s}}$ scale for shower splittings. In this way, we match the $\alpha_{\mathrm{S}}$ settings used in the DEDUCTOR shower. Of course, PYTHIA and DEDUCTOR use very different algorithms to generate their parton showers. Of particular note are the inclusion of a summation of threshold logarithms in DEDUCTOR and the differences between PYTHIA and DEDUCTOR in splitting functions, shower ordering variable, and momentum mapping at each splitting. Thus we can expect only rough agreement between the programs. Nevertheless, it is of interest to see how much disagreement there is.

We exhibit the comparison in Fig. 8. We see that the differences between PYTHIA and DEDUCTOR with the LC + approximation are fairly modest except at the largest values of $y_{12}$, for which it appears that PYTHIA produces more initial state radiation that can destroy the gap and thus make the gap fraction smaller.

\section{CONCLUSIONS}

DEDUCTOR is a parton shower event generator whose primary purpose is to implement new theoretical developments in parton shower algorithms so as to improve the precision and predictive power of parton shower event generators. In a general framework [34], a parton shower is a solution of a renormalization group equation in which the generators of scale changes are, at order $\alpha_{\mathrm{s}}$, operators called $\mathcal{H}_{I}(t)$ and $\mathcal{V}(t)$ in DEDUCTOR.

One of the important questions in this program is how to deal with color in the shower evolution. Color in parton showers has typically been treated in the leading color (LC) approximation. Previous versions of DEDUCTOR used the $\mathrm{LC}+$ approximation, which is an improvement over the $\mathrm{LC}$ approximation but is nowhere near exact: $\mathcal{H}_{I}(t)$ and $\mathcal{V}(t)$

\footnotetext{
${ }^{2}$ DEDUCTOR itself includes only a parton shower based on perturbative splitting functions. However, on can, if desired, add an underlying event as contained in a nonperturbative model and then send the resulting partons to PYTHIA for hadronization.[10].
}

contain contributions $\Delta \mathcal{H}(t)$ and $\Delta \mathcal{V}(t)$ that are simply dropped in the LC+ approximation. The current version of DEDUCTOR [11] allows one to include $\Delta \mathcal{H}(t)$ and $\Delta \mathcal{V}(t)$ perturbatively. That is, a limited number of powers of $\Delta \mathcal{H}(t)$ and $\Delta \mathcal{V}(t)$ can be included in a calculation.

In Ref. [11], we found that a calculation beyond the $\mathrm{LC}+$ approximation could give numerical results for a physical cross section. We chose the one-jet-inclusive cross section and found an approximately 3\% effect from the extra color. In the present paper, we have asked whether DEDUCTOR with improved color can produce numerical results for more complicated physical cross sections and whether there are examples in which the effects of extra color are numerically at a level of $1 / N_{\mathrm{c}}^{2} \sim 10 \%$ or higher.

We have chosen as our example the rapidity gap survival probability. This observable is of some practical significance because its study can help us to understand the effect in a search for new physics signals of vetoing against extra jets with transverse momenta greater than a cutoff $p_{\mathrm{T}}^{\text {cut }}$. It is also of special interest because it contains nonglobal logarithms, which are not simple to sum in an analytical approach.

We found in this study that the effects of extra color are substantial for soft gluon emissions when the rapidity difference, $y_{12}$, between the two leading jets and the average, $\bar{p}_{\mathrm{T}}$, of their transverse momenta are large. We also found that the contribution of $\mathrm{i} \pi$ terms in $\Delta \mathcal{V}(t)$ saturates and does not grow significantly with the rapidity separation. It is interesting that these effects work into the opposite directions. The wide angle soft gluon emissions decrease the survival rate while the $\mathrm{i} \pi$ terms increase it. Finally, we found that kinematic effects that result from exactly conserving momentum in the parton shower are numerically important.

Since we included the $\Delta \mathcal{H}(t)$ and $\Delta \mathcal{V}(t)$ only perturbatively, one can ask how many powers of these operators we need to make stable predictions. We tested this and we found that, for the gap fraction, the result is rather stable after two insertions of the soft correction operators. This finding is important because we cannot actually include many powers of $\Delta \mathcal{H}(t)$ and $\Delta \mathcal{V}(t)$. First, we cannot simply work to all orders in these operators. The dimension of the color space grows roughly as $(N !)^{2}$ with the number of partons. In a typical shower calculation the averaged number of partons is $20-30$. It is clear that there is no hope to deal with this problem exactly, so that one must use a perturbative approach. In the perturbative calculation we cannot include a very large power of the soft correction operators because the computer resource demand of the program gets out of control very quickly.

Parton showers have their own systematic logic as operator renormalization group evolution [34] in which, so far, we know only the order $\alpha_{\mathrm{s}}$ contributions to the generators of scale changes. Using a parton shower to calculate an observable like the gap fraction $f$ has the effect of summing large logarithms. With a loose interpretation of 
what constitutes a logarithm, there are three sorts of large logarithms $L$ in $f: \log \left(\bar{p}_{\mathrm{T}} / p_{\mathrm{T}}^{\text {cut }}\right), y_{12}$, and $\mathrm{i} \pi$. Then in perturbation theory we have contributions $\alpha_{\mathrm{s}}^{n} L^{k}$ with $k \leq 2 n$. One might hope that $\log f$ has an expansion with terms $\alpha_{\mathrm{s}}^{n} L^{k}$ with $k \leq n+1$ in both full QCD and in an all orders parton shower and that a leading order parton shower gets the $\alpha_{\mathrm{s}} L^{2}$ and $\alpha_{\mathrm{s}} L$ contributions to $\log f$ correctly. However, a proof of this conjecture would not be easy and lies beyond the scope of this paper.

The calculation presented here does not include matching to NLO perturbation theory. In its current version, DEDUCTOR starts with the color density matrix for $2 \rightarrow 2$ QCD scattering at order $\alpha_{\mathrm{s}}^{2}$. It then applies an operator $\mathcal{U}_{\mathcal{V}}$ that sums threshold logarithms, as described in Ref. [10]. After that, it applies the operator $\mathcal{U}$ that generates parton splittings and thus a parton shower. Imagine expanding the combined operator $\mathcal{U} \mathcal{U}_{\mathcal{V}}$ in powers of $\alpha_{\mathrm{s}}$. The first term is simply the unit operator, but then there are terms proportional to $\alpha_{\mathrm{s}}^{1}$ and higher powers of $\alpha_{\mathrm{s}}$. The term proportional to $\alpha_{\mathrm{s}}^{1}$, multiplying the $\alpha_{\mathrm{s}}^{2}$ hard scattering color density matrix, gives an approximation to the order $\alpha_{\mathrm{s}}^{3}$ density matrix. It is an approximation because the operators $\mathcal{U}$ and
$\mathcal{U}_{v}$ are based on soft and collinear limits. As explained in Ref. [34], it is possible to include the complete order $\alpha_{\mathrm{s}}^{3}$ color density matrix while correcting for the $\alpha_{\mathrm{s}}^{3}$ contributions provided by $\mathcal{U}$ and $\mathcal{U}_{\mathcal{V}}$. The procedure for this matching is straightforward, although it is more complex than the procedure needed to match cross sections because the object that needs matching is the color density matrix rather than just its trace over color. With NLO matching, calculations like the gap fraction calculation presented here would be more accurate and less sensitive to scale parameter choices such as the choice of the hardness scale at which the shower starts. We hope to add NLO matching in a future version of DEDUCTOR.

\section{ACKNOWLEDGMENTS}

This work was supported in part by the United States Department of Energy under Grant No. DE-SC0011640. This work benefited from access to the University of Oregon high performance computer, Talapas, and from access to the DESY Theory Group computer cluster.
[1] Z. Nagy and D. E. Soper, Parton showers with quantum interference, J. High Energy Phys. 09 (2007) 114.

[2] Z. Nagy and D. E. Soper, Parton showers with quantum interference: Leading color, spin averaged, J. High Energy Phys. 03 (2008) 030.

[3] Z. Nagy and D. E. Soper, Parton showers with quantum interference: Leading color, with spin, J. High Energy Phys. 07 (2008) 025.

[4] Z. Nagy and D. E. Soper, Parton shower evolution with subleading color, J. High Energy Phys. 06 (2012) 044.

[5] Z. Nagy and D. E. Soper, A parton shower based on factorization of the quantum density matrix, J. High Energy Phys. 06 (2014) 097.

[6] Z. Nagy and D. E. Soper, Ordering variable for parton showers, J. High Energy Phys. 06 (2014) 178.

[7] Z. Nagy and D. E. Soper, Parton distribution functions in the context of parton showers, J. High Energy Phys. 06 (2014) 179.

[8] Z. Nagy and D. E. Soper, Effects of subleading color in a parton shower, J. High Energy Phys. 07 (2015) 119.

[9] Z. Nagy and D. E. Soper, Summing threshold logs in a parton shower, J. High Energy Phys. 10 (2016) 019.

[10] Z. Nagy and D. E. Soper, Jets and threshold summation in Deductor, Phys. Rev. D 98, 014035 (2018).

[11] Z. Nagy and D. E. Soper, Parton showers with more exact color evolution, Phys. Rev. D 99, 054009 (2019).

[12] M. Cacciari, G. P. Salam, and G. Soyez, The anti-k(t) jet clustering algorithm, J. High Energy Phys. 04 (2008) 063.
[13] J. Forshaw, J. Keates, and S. Marzani, Jet vetoing at the LHC, J. High Energy Phys. 07 (2009) 023.

[14] N. Kidonakis, G. Oderda, and G. F. Sterman, Evolution of color exchange in QCD hard scattering, Nucl. Phys. B531, 365 (1998).

[15] G. Oderda and G. F. Sterman, Energy and Color Flow in Dijet Rapidity Gaps, Phys. Rev. Lett. 81, 3591 (1998).

[16] J. R. Forshaw, A. Kyrieleis, and M. H. Seymour, Gaps between jets in the high energy limit, J. High Energy Phys. 06 (2005) 034.

[17] M. Dasgupta and G. P. Salam, Resummation of nonglobal QCD observables, Phys. Lett. B 512, 323 (2001).

[18] C. F. Berger, T. Kucs, and G. F. Sterman, Energy flow in interjet radiation, Phys. Rev. D 65, 094031 (2002).

[19] M. Dasgupta and G. P. Salam, Accounting for coherence in interjet $E(t)$ flow: A case study, J. High Energy Phys. 03 (2002) 017.

[20] R. B. Appleby and M. H. Seymour, Nonglobal logarithms in interjet energy flow with kt clustering requirement, J. High Energy Phys. 12 (2002) 063.

[21] J. R. Forshaw, A. Kyrieleis, and M. H. Seymour, Superleading logarithms in non-global observables in QCD?, J. High Energy Phys. 08 (2006) 059.

[22] J. R. Forshaw, A. Kyrieleis, and M. H. Seymour, Superleading logarithms in non-global observables in QCD: Colour basis independent calculation, J. High Energy Phys. 09 (2008) 128.

[23] R. M. Duran Delgado, J. R. Forshaw, S. Marzani, and M. H. Seymour, The dijet cross section with a jet veto, J. High Energy Phys. 08 (2011) 157. 
[24] J. R. Andersen and J. M. Smillie, Multiple jets at the LHC with high energy jets, J. High Energy Phys. 06 (2011) 010.

[25] S. Alioli, J. R. Andersen, C. Oleari, E. Re, and J. M. Smillie, Probing higher-order corrections in dijet production at the LHC, Phys. Rev. D 85, 114034 (2012).

[26] T. Sjöstrand, S. Ask, J. R. Christiansen, R. Corke, N. Desai, P. Ilten, S. Mrenna, S. Prestel, C. O. Rasmussen, and P. Z. Skands, An introduction to PYTHIA 8.2, Comput. Phys. Commun. 191, 159 (2015).

[27] M. Bahr et al., Herwig++ physics and manual, Eur. Phys. J. C 58, 639 (2008).

[28] T. Gleisberg, S. Hoeche, F. Krauss, M. Schonherr, S. Schumann, F. Siegert, and J. Winter, Event generation with SHERPA 1.1, J. High Energy Phys. 02 (2009) 007.
[29] R. Ángeles Martínez, M. De Angelis, J. R. Forshaw, S. Plätzer, and M. H. Seymour, Soft gluon evolution and non-global logarithms, J. High Energy Phys. 05 (2018) 044.

[30] S. Plätzer and M. Sjödahl, Subleading $N_{c}$ improved parton showers, J. High Energy Phys. 07 (2012) 042.

[31] J. Isaacson and S. Prestel, Stochastically sampling color configurations, Phys. Rev. D 99, 014021 (2019).

[32] S. Plätzer, M. Sjödahl, and J. Thorén, Color matrix element corrections for parton showers, J. High Energy Phys. 11 (2018) 009.

[33] Z. Nagy, Next-to-leading order calculation of three jet observables in hadron hadron collision, Phys. Rev. D 68, 094002 (2003).

[34] Z. Nagy and D. E. Soper, What is a parton shower?, Phys. Rev. D 98, 014034 (2018). 